\title{
Arteriographic patterns early in the onset of the coronary syndromes ${ }^{1}$
}

\author{
Valentin Fuster, Robert L. Frye, Daniel C. Connolly, Margaret A. Danielson, \\ Lila R. Elveback, and Leonard T. Kurland \\ From the Division of Cardiovascular Diseases, and the Department of Medical Statistics and Epidemiology, \\ Mayo Clinic and Mayo Foundation, Rochester, Minnesota, U.S.A.
}

Coronary arteriography in 300 patients within one year of onset of symptoms of coronary arterial disease revealed already severe anatomical coronary disease in three patient groups: those with angina pectoris alone (164 patients), with subendocardial myocardial infarction (63 patients), and with transmural myocardial infarction (73 patients). The number of vessels diseased ( $\geqslant 50 \%$ obstruction), distribution of obstruction, and degree of stenosis were similar in the three groups. However, total occlusion of at least one artery was much more common in transmural myocardial infarction and in subendocardial myocardial infarction with elevation of enzyme levels. We suggest that such occlusions occurred at the time of the infarction. Similarities in coronary anatomy between patient subgroups with angina (on exercise or at rest and nocturnal) indicate that factors other than coronary anatomy intervene in precipitating the different types of angina. Vessel disease was not related to smoking, hyperlipidaemia, or hypertension but coronary disease was manifest earlier in life in smokers or those with hyperlipidaemia.

There is little information about the differences in arteriographic patterns among three of the syndromes of coronary artery disease: angina pectoris, subendocardial myocardial infarction, and transmural myocardial infarction. Such paucity of information may be explained by the fact that patients with coronary artery disease often undergo arteriographic studies at an advanced stage of the diseasefor example, manifestations of coronary artery disease may have persisted for several years or the different coronary syndromes may have already occurred in the same patient for a long period before the study. Accordingly, the present study was designed to document the clinical-arteriographic correlations of the three specific coronary syndromes at an early stage-within one year of the initial manifestation of coronary artery disease. We also sought to determine whether cigarette smoking, hyperlipidaemia, or hypertension exert some influence on the coronary anatomy at this early stage of clinically manifested coronary artery disease.

Received 21 April 1975.

This investigation was supported in part by a Research Grant from the National Institutes of Health, Public Health Service, Bethesda, Maryland, U.S.A.

\section{Patients and methods}

\section{Clinical groups}

The study group comprised 300 patients $(265$ men and 35 women) who were seen consecutively at the Mayo Clinic from July 1968 to June 1973 and who had an arteriographic study of satisfactory quality within one year of onset of typical symptoms of coronary artery disease. Patients who had congenital or rheumatic heart disease were not included. There were also 5 patients with classical angina pectoris and 1 patient with transmural myocardial infarction who had normal coronary arteriograms and were excluded from the study. All patients' histories were taken by at least three physicians and the clinical records were carefully examined in retrospect by one of us (V.F.) who had no knowledge of the arteriographic findings. Additional information when needed was obtained from letters and telephone calls to the referring physicians. The patients were divided into three clinical groups.

Group 1 Patients with angina pectoris alone (164 patients). Group 1 was further divided into two subgroups, group 1A and group 1B. Group 1A comprised 100 patients in whom substernal or praecordial pain or discomfort was precipitated by walking (and relieved within 15 minutes by rest), stress, or excitement. Group 1B comprised 64 patients in whom pain occurred at rest or at night, lasted less than 15 minutes, and was similar to that brought on by physical exertion. Among 
the 64 patients in group 1B 18 had angina that was considered unstable-that is, angina at rest or at night occurring with an increase in intensity, duration, or frequency of pain within three weeks before the study. None of the patients in group 1 had evidence of transmural or subendocardial myocardial infarction.

Group 2 Patients with subendocardial myocardial infarction (63 patients). Patients in this group had a typical history of acute myocardial infarction and ST-T changes only in the electrocardiogram fulfilling the criteria of the Coronary Drug Project (1973)-that persisted for more than 48 hours. Group 2 was further divided into two subgroups: group $2 \mathrm{~A}(68 \%)$ who did not have a diagnostic increase in enzyme levels at the time of the infarction, and group $2 \mathrm{~B}(32 \%)$ who had a diagnostic increase in enzyme levels. About two-thirds of all patients had had angina pectoris before the subendocardial infarct (but in all instances the angina occurred less than one year before the arteriographic study) and most ( $87 \%$ ) had angina pectoris after the subendocardial infarction before the arteriographic study.

Group 3 Patients with transmural myocardial infarction (73 patients). In this group of patients the clinical history was suggestive of acute myocardial infarction, with definite QRS changes in the ECGfulfilling the criteria of the Coronary Drug Project (1973)-and diagnostic rise in serum enzyme levels. About one-fourth of these patients had angina pectoris before the infarct (but in all instances the angina occurred less than one year before the arteriographic study) and about two-thirds had angina pectoris after the infarct and before the arteriographic study.

\section{Evaluation of coronary risk factors}

Each patient was evaluated for the possible influence on coronary artery disease of smoking, hyperlipidaemia, and hypertension.

Cigarette smoking Patients were subdivided into three categories: (1) those who smoked more than 10 a day (172 patients $(60 \%))$; (2) those who had smoked in the past but who had stopped smoking more than six months before the initial manifestation of coronary artery disease ( 54 patients $(18 \%)$ ); and (3) those who did not smoke cigarettes ( 71 patients $(24 \%)$ ). The smoking habits were not recorded for three patients.

Hyperlipidaemia Patients were considered to be hyperlipidaemic (61 patients $(20 \%)$ ) if a diagnosis of hypertriglyceridaemia or hypercholesterolaemia was made before the diagnosis of coronary disease (even though such patients were subsequently given lipid-lowering medication) or if, at the time of admission for coronary arteriography, the serum cholesterol concentration was $7.8 \mathrm{mmol} / 1(300 \mathrm{mg} / \mathrm{dl})$ or more or the serum triglyceride concentration was $1.7 \mathrm{mmol} / 1(150 \mathrm{mg} / \mathrm{dl})$ or more in a 14-hour fasting specimen.

Hypertension Patients were considered to have definite high blood pressure (52 patients $(17 \%)$ ) if diagnosis of hypertension had been made before the diagnosis of coronary artery disease (even though such patients were subsequently given antihypertensive medication) or if, at the time of admission for coronary arteriography, the average systolic pressure, recorded on three separate occasions, was $160 \mathrm{mmHg}(21.3 \mathrm{kPa})$ or more, or the diastolic pressure averaged $95 \mathrm{mmHg}$ $(12.6 \mathrm{kPa})$ or more. If the average systolic pressure was 140 to $159 \mathrm{mmHg}(18.6$ to $21.1 \mathrm{kPa})$ or if the average diastolic pressure was 90 to $94 \mathrm{mmHg}(12.0$ to $12.5 \mathrm{kPa}$ ) a patient was considered to have borderline hypertension (33 patients $(11 \%)$ ).

\section{Coronary arteriography}

Selective coronary arteriography was performed in all patients by the Sones and Shirey (1962) technique. The arteriograms were viewed initially for diagnostic purposes and reviewed later by one of us (R.L F.) without knowledge of the patients' clinical or haemodynamic data.

Multiple coronary arteriographic views in different oblique projections were studied and the degree of obstruction in a vessel was derived from estimates of the width of the respective vessel in its normal portion compared to its most diseased portion, the latter being expressed as a percentage of the former. Obstruction only of at least 50 per cent of one or more of the four major arteries-right coronary, left anterior descending coronary, left circumflex, and left main coronary artery or their main branches-was considered. For the purposes of this study four degrees of obstruction were considered: 50 to 74 per cent, 75 to 89 per cent, 90 per cent to subtotal, and occlusion.

The number of diseased vessels in each patient was determined by obstruction of one, two, or three major arteries-right coronary, left anterior descending coronary, and left circumflex artery. Lesions of the left main coronary artery, alone or in combination with lesions in the other arteries, were considered separately. The presence of intercoronary collateral circulation, visualized as a retrograde filling of a diseased coronary artery, was also noted and recorded.

\section{Results}

\section{Clinical groups and arteriographic patterns}

Number of vessels involved About one-third $(31 \%)$ of the patients in group 1 had disease in one vessel, about one-third (37\%) had disease in two vessels, and about one-third ( $32 \%)$ had either disease in three vessels or a lesion in the left main coronary artery (Fig. 1). The findings were similar in patients in groups 2 and 3 (Fig. 1). Among patients in groups 2 and 3 the presence or absence of angina pectoris before or after the infarct was not associated with much arteriographic variation. In the entire group of 300 patients the average number of vessels diseased per patient was two. Since the degree of obstruction was greater than 75 per cent in all patients except four, in whom only one vessel was diseased, disease of the coronary arteries was usually severe when symptoms first developed. 
Number of vessels involved
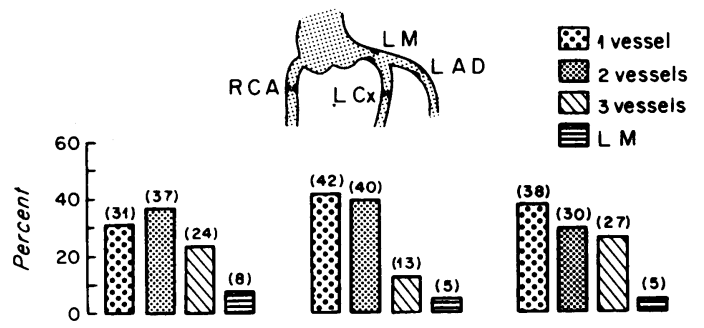

Distribution of vessel involvement

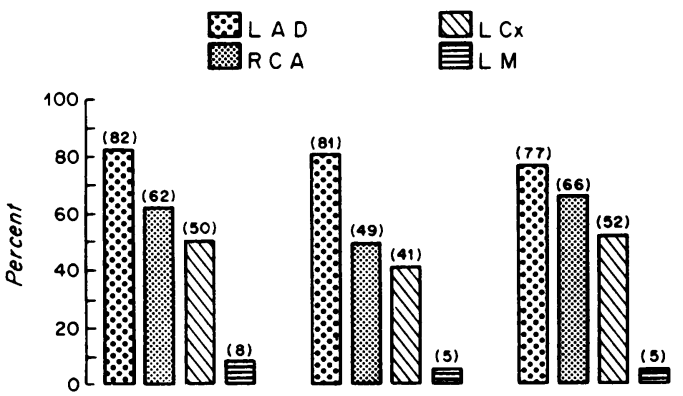

Degree of vessel obstruction

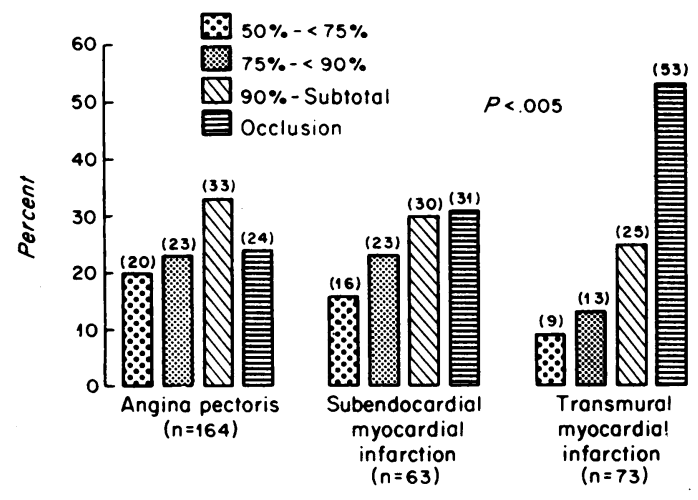

FIG. 1 Arteriographically demonstrated number, distribution, and degree of vessels diseased in patients with angina pectoris, subendocardial myocardial infarction, and transmural myocardial infarction. Number and distribution of vessels diseased are expressed as percentage of patients with each clinical syndrome. Degree of vessel obstruction is expressed as percentage of vessels diseased in each clinical syndrome. $L A D=$ left anterior descending coronary artery; $R C A=$ right coronary artery; $L C x=$ left circumflex coronary artery; $L M=$ left main coronary artery, which is considered alone or in combination with lesions in other arteries.
Distribution of vessel involvement The location of arterial obstruction was also strikingly similar in patients in the three main groups (Fig. 1). The left anterior descending coronary artery was the vessel most often affected-in 82 per cent of patients in group 1,81 per cent in group 2, and in 77 per cent in group 3. The right coronary artery, left circumflex artery, and left main coronary artery, were less frequently affected in that order. Among patients in groups 2 and 3 the presence or absence of angina pectoris before or after the infarct was not associated with any arteriographic variation.

Degree of vessel obstruction With respect to the total number of vessels diseased the proportion of vessels with each degree of stenosis did not differ greatly in the three groups of patients (Fig. 1), but the percentage of completely occluded vessels was significantly $(P<0.001)$ higher in patients with transmural myocardial infarction (Fig. 1) and in patients with subendocardial myocardial infarction who had an increase in enzyme levels (Table).

TABLE Number of patients with one or more vessels occluded in relation to clinical diagnosis

\begin{tabular}{|c|c|c|}
\hline \multirow{3}{*}{$\begin{array}{l}\text { Diagnosis } \\
\text { Angina pectoris (group 1) } \\
\text { Subendocardial myocardial infarction } \\
\text { (group } 2 \mathrm{~A})\end{array}$} & \multicolumn{2}{|c|}{$\begin{array}{l}\text { Patients } \\
\text { No. }(\%)\end{array}$} \\
\hline & $69 / 164$ & (42) \\
\hline & $16 / 43$ & (37) \\
\hline $\begin{array}{l}\text { Subendocardial myocardial infarction } \\
\text { (group 2B) }\end{array}$ & $14 / 20$ & $(70)^{*}$ \\
\hline $\begin{array}{l}\text { Transmural myocardial infarction } \\
\text { (group 3) }\end{array}$ & $58 / 73$ & $(79)^{*}$ \\
\hline
\end{tabular}

*Significantly different $(P<0.001)$ from percentages for the other two groups.

\section{Factors precipitating angina pectoris and arteriographic patterns}

To determine whether patients with angina at rest or at night had a more advanced anatomical stage of coronary artery disease than those with only external and emotional angina, we compared the number, distribution, and degree of vessels diseased in both subgroups of patients in group 1 (Fig. 2). In these respects the two subgroups were nearly identical. The presence or absence of collateral circulation was also similar in both groups. Furthermore, the patients with unstable angina pectoris manifested no differential arteriographic features when these were compared with the features noted in the other anginal groups, with three exceptionsa slight increase in incidence of one-vessel disease $(P<0.05)$, a decrease in incidence of vessels with total occlusion $(P<0.05)$, and a decrease of collateral supply $(P<0 \cdot 05)$. 

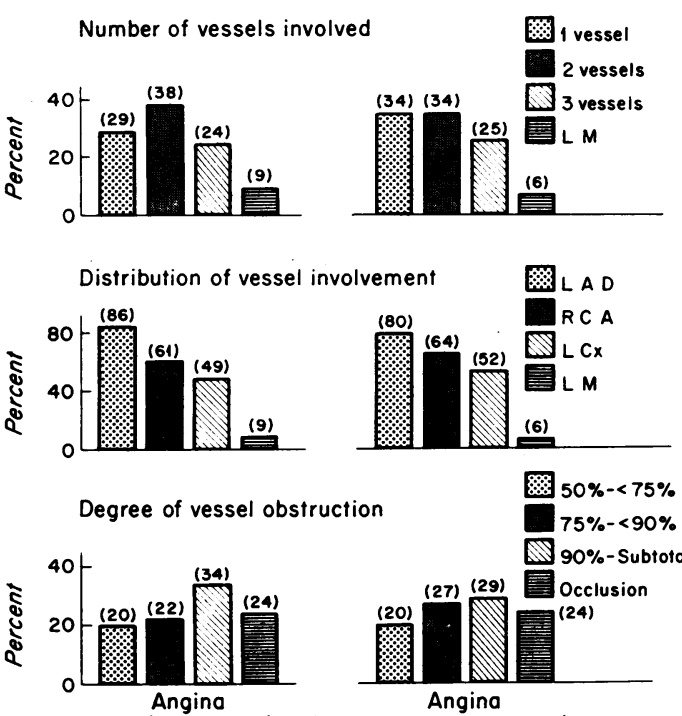

on exercise or emotional $(n=100)$

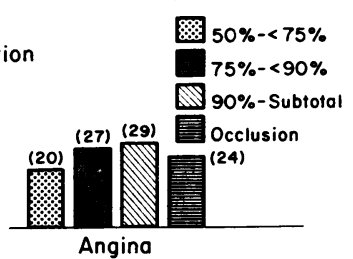

at rest or nocturnal $(n=64)$

FIG. 2 Arteriographically demonstrated number, distribution, and degree of vessels diseased related to factors precipitating angina pectoris. For explanation of calculation of number, distribution, and degree of vessel disease and of abbrevations see legend to Fig. 1 .

\section{Coronary risk factors and arteriographic patterns}

Cigarette smoking The number, distribution, and degree of vessels diseased were similar in patients who had never smoked and in those who had smoked before or at the time of the initial manifestation of coronary artery disease (Fig. 3). Furthermore, no arteriographic differences were apparent when smokers were considered in two classesthose who smoked at the time of the initial manifestation of coronary artery disease and those who had smoked in the past but had stopped more than six months before the initial manifestation of coronary artery disease. Analysis of the age distribution, however, showed that smokers had clinical manifestations of coronary artery disease significantly earlier in life than non-smokers (Fig. 4).

Hyperlipidaemia When patients with hyperlipidaemia were compared with patients with normal serum lipid concentrations the number, distribution, and degree of vessels diseased were found to be similar (Fig. 5). Analysis of age distribution, however, showed that patients with hyperlipidaemia developed clinical manifestations of coronary artery disease at a younger age than did patients with normal lipids (Fig. 4).

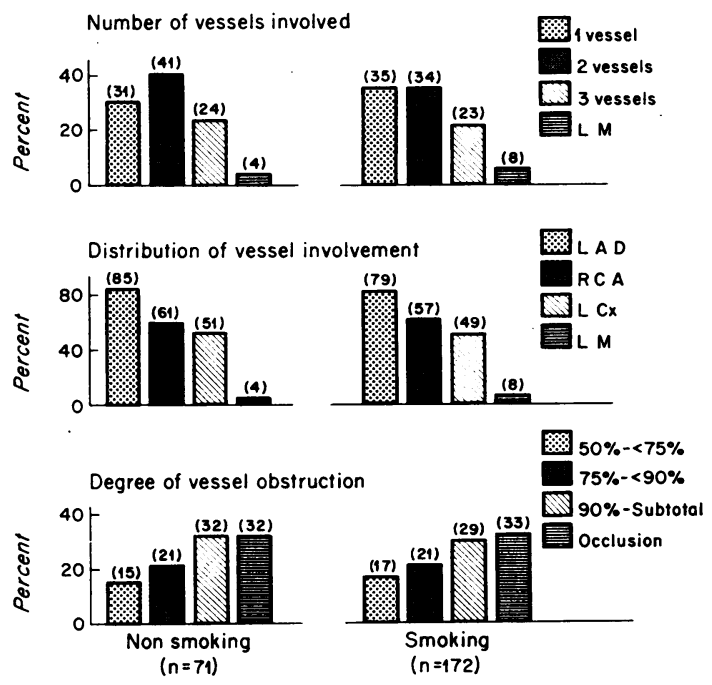

FIG. 3 Arteriographically demonstrated number, distribution, and degree of vessels diseased related to patients' smoking habits. For explanation of calculation of number, distribution, and degree of vessel disease and of abbreviations see legend to Fig. 1.

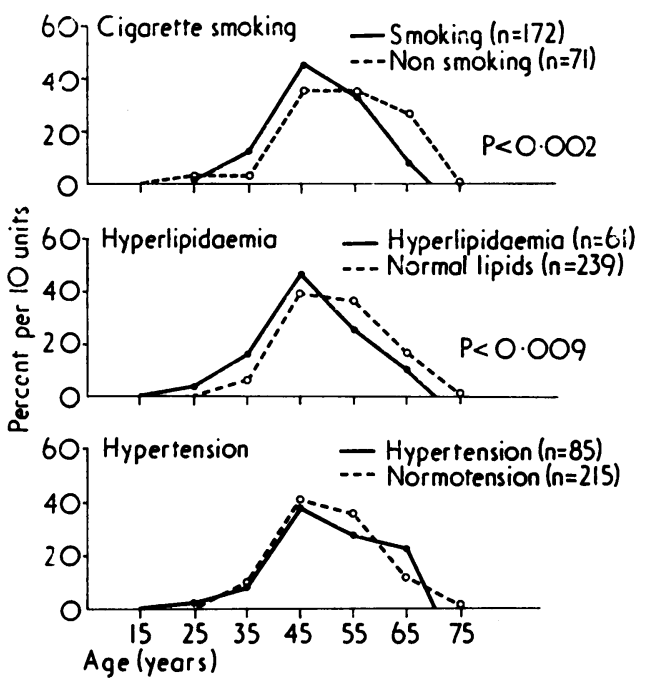

FIG. 4 Age distribution analysis relating age at first clinical manifestation of coronary artery disease to cigarette smoking or non-smoking, hyperlipidaemia, and hypertension.

Hypertension The number, distribution, and degree of vessels diseased were similar in patients with hypertension and in the normotensive group 

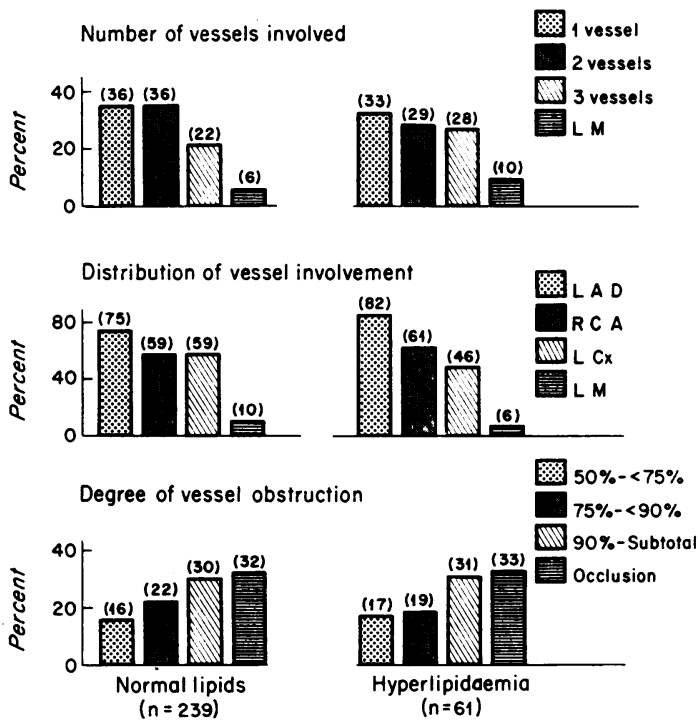

FIG. 5 Arteriographically demonstrated number, distribution, and degree of vessels diseased related to patients' serum lipid concentrations. For explanation of calculation of number, distribution, and degree of vessel disease and of abbreviations see legend to Fig. 1.
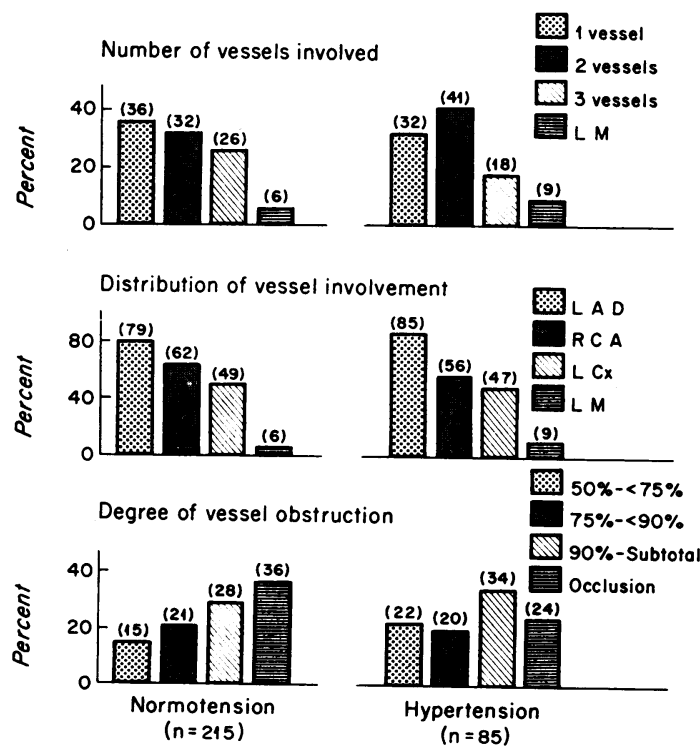

FIG. 6 Arteriographically demonstrated number, distribution, and degree of vessels diseased related to patients' blood pressures. For explanation of calculation of number, distribution, and degree of vessel disease and of abbreviations see legend to Fig. 1.
(Fig. 6). Furthermore, the findings were similar when the hypertensive patients were subdivided into those classified as having definite high blood pressure and those defined as having borderline hypertension. There was no difference in age distribution at onset of coronary artery disease between the hypertensive and the normotensive group (Fig. 4). Stepwise discriminant analysis revealed that no combination of the three coronary risk factors had any influence on the arteriographic patterns.

\section{Discussion}

The present large series of patients with coronary arterial disease differs from other series because we selected only those patients who had had an arteriographic study at an early stage-within one yearof the onset of symptoms of coronary artery disease. This allowed us to determine accurately the initial manifestations of the disease and to classify precisely and independently the different coronary syndromes before further evolution of the disease.

Our findings suggest that coronary arterial disease is usually severe when the first clinical manifestation develops. Of the 300 patients who were studied about two-thirds had disease of two or three vessels or a lesion in the left main coronary artery, and only in four patients was the disease confined to one vessel with less than 75 per cent obstruction. This observation of the severity of disease of the coronary arteries in the early stage of clinically manifested coronary artery disease has been suggested by the previous studies of Proudfit et al. (1968) and Parker, Di Giorgi, and West (1966), but the number of patients was small and they were not clinically characterized according to their type of coronary syndrome.

The groups of patients with angina pectoris, subendocardial myocardial infarction, or transmural myocardial infarction were similar with respect to the number and distribution of diseased arteries. However, analysis of the degree of disease of vessels showed that the incidence of total occlusion of at least one major artery was much higher in patients who had transmural myocardial infarction or subendocardial myocardial infarction with increase in enzyme levels than it was in the other groups of patients. This finding together with our observations that arterial occlusions in patients with transmural myocardial infarction were proximal to the location of the infarct as defined by electrocardiogram and ventriculogram (Fuster et al., 1974b) supports the thesis that anatomical features of the coronary arteries in acute transmural myocardial infarction differ from those 
in angina pectoris in that one of the arteries previously incompletely obstructed becomes occluded, presumably suddenly. The possibility that in some instances this arterial occlusion is a secondary event rather than the cause of the infarct has been raised by the clinicopathological studies of Roberts (1972). The fewer completely occluded vessels in patients who had angina pectoris probably reflect the result of a disease that is only slowly progressive, permitting the development of collateral circulation. This possibility is supported by the high incidence of a collateral blood supply in association with occluded vessels in patients with angina pectoris as compared with those with transmural myocardial infarction (Fuster et al., 1974a).

The observation that arteriographic patterns in patients with angina at rest or at night were similar to those in patients with only exertional and emotional angina tends to support the clinical impression that factors other than anatomical ones seem also to play a role in the precipitation of the syndrome of angina pectoris.

Our angiographic data indicate that when the first clinical manifestations of coronary artery disease appear the presence or absence of cigarette smoking, hyperlipidaemia, and hypertension was not correlated with the coronary anatomical patterns. The first clinical manifestations of coronary artery disease seem to be caused when there was an advanced degree of anatomical disease irrespective of whether those risk factors were present or not. Among cigarette smokers and patients with hyperlipidaemia, however, manifestations of coronary artery disease did develop at an earlier stage in life than among non-smokers and patients with normal concentrations of lipids. It has also been shown that cigarette smoking, high serum cholesterol values, and hypertension strikingly increase the incidence rate of coronary artery disease (Atherosclerosis and Epidemiology Study Groups, 1970), but whether such risk factors accelerate the progress of the coronary disease is controversial (Bemis et al., 1973; Bruschke,
Proudfit, and Sones, 1973; Frank, Weinblatt, and Shapiro, 1973; Oxman et al., 1973).

We thank Miss Joan M. Rohlik for her valuable assistance.

\section{References}

Atherosclerosis and Epidemiology Study Groups (1970). Primary prevention of the atherosclerotic diseases. Circulation, 42, A55.

Bemis, C. E., Gorlin, R., Kemp, H. G., and Herman, M. V. (1973). Progression of coronary artery disease: a clinical arteriographic study. Circulation, 47, 455.

Bruschke, A. V. G., Proudfit, W. L., and Sones, F. M., Jr. (1973). Progress study of 590 consecutive nonsurgical cases of coronary disease followed 5-9 years. II. Ventriculographic and other correlations. Circulation, 47, 1154.

Coronary Drug Project (1973). Design, methods and baseline results. Circulation, 47, Suppl 1, 1-39.

Frank, C. W., Weinblatt, E., and Shapiro, S. (1973). Angina pectoris in men: prognostic significance of selected medical factors. Circulation, 47, 509.

Fuster, V., Connolly, D. C., Frye, R. L., Danielson, M. A., Elveback, L. R., and Kurland, L. T. (1974a). Angiographic patterns early in the onset of the coronary syndromes (abstract). American fournal of Cardiology, 33, 138.

Fuster, V., Frye, R. L., Connolly, D. C., Danielson, M. A., and Mankin, H. T. (1974b). Electrocardiographic-angiographic correlation and its influence by collaterals early in the onset of the coronary syndromes (abstract). Circulation, 50, Suppl 3, III-108.

Oxman, H. A., Connolly, D. C., Nobrega, F. T., and Titus, J. L. (1973). Identification of the patients at highest risk for sudden death within five years following their first myocardial infarction (abstract). American fournal of Cardiology, 31, 150.

Parker, J. O., Di Giorgi, S., and West, R. O. (1966). Selective coronary arteriography: arteriographic patterns in coronary heart disease. Canadian Medical Association fournal, 95, 291.

Proudfit, W. L., Shirley, E. K., Sheldon, W. C., and Sones, F. M., Jr. (1968). Certain clinical characteristics correlated with extent of obstructive lesions demonstrated by selective cine-coronary arteriography. Circulation, 38, 947.

Roberts, W. C. (1972). Coronary arteries in fatal acute myocardial infarction. Circulation, 45, 215.

Sones, F. M., Jr., and Shirley, E. K. (1962). Cine coronary arteriography. Modern Concepts of Cardiovascular Disease, 31, 735.

Requests for reprints to Dr. Valentin Fuster, Division of Cardiovascular Diseases, Mayo Clinic, 200 First Street SW, Rochester, Minnestoa 55901, U.S.A. 